Research Paper

\title{
Long Noncoding RNA FAM225A Promotes Esophageal Squamous Cell Carcinoma Development and Progression via Sponging MicroRNA-197-5p and Upregulating NONO
}

Pengyuan Zhu' ${ }^{1}$, Haitao Huang ${ }^{2}$, Shaorui $\mathrm{Gu}^{2}$, Zhenchuan $\mathrm{Liu}^{2}$, Xin Zhang ${ }^{2}$, Kaiqin $\mathrm{Wu}^{2}$, Tiancheng $\mathrm{Lu}^{2}$, Lei $\mathrm{Li}^{2}$, Chenglai Dong${ }^{2}$, Chongjun Zhong ${ }^{\circledR}$, Yongxin Zhou ${ }^{2}$

1. Department of thoracic and Cardiovascular Surgery, the Second Affiliated Hospital of Nantong University, School of Medicine, Nantong University, Nantong, 226001, China.

2. Department of thoracic and Cardiovascular Surgery, Tongji Hospital, Tongji University School of Medicine, Shanghai, 200065, China.

$\triangle$ Corresponding author: Chongjun Zhong, zhongcjnt001@126.com, Department of thoracic and Cardiovascular Surgery, the Second Affiliated Hospital of Nantong University, School of Medicine, Nantong University, Nantong, 226001, China. Yongxin Zhou, zhou6302@tongii.edu.cn, Department of thoracic and Cardiovascular Surgery, Tongji Hospital, Tongji University School of Medicine, Shanghai, 200065, China.

(c) The author(s). This is an open access article distributed under the terms of the Creative Commons Attribution License (https://creativecommons.org/licenses/by/4.0/). See http://ivyspring.com/terms for full terms and conditions.

Received: 2020.07.30; Accepted: 2020.11.16; Published: 2021.01.01

\begin{abstract}
Esophageal squamous cell carcinoma (ESCC) is the major subclass of esophageal cancer and one of the most life-threatening malignancies with high morbidity and mortality. Long noncoding RNAs (IncRNAs) participate in tumorigenesis and metastasis of various tumors. Here, we investigated the function of a newly identified IncRNA FAM225A in ESCC. LncRNA FAM225A expression was significantly higher in ESCC and predicted poor prognosis of ESCC patients. We confirmed that upregulation of FAM225A in ESCC and overexpression of FAM225A was associated with poor outcome in ESCC patients using TCGA ESCC cohort. Knockdown of FAM225A significantly inhibited cell growth, migration and invasion of ESCC cells in vitro and inhibited ESCC xenograft development in vivo. Mechanistically, we demonstrated that IncRNA FAM225A functioned as a competing endogenous RNA (ceRNA) via sponging miR-197-5p. LncRNA FAM225A exerted its regulatory function on ESCC proliferation and metastasis via modulating expression of miR-197-5p. MiR-197-5p overexpression antagonized the function of FAM225A, with decreased cell growth and invasion. Moreover, we identified that RNA binding protein NONO was a direct target of miR-197-5p and miR-197-5p negatively regulated NONO expression and TGF- $\beta$ signaling in ESCC cells. In summary, our findings suggest that IncRNA FAM225A promotes ESCC development and progression via sponging miR-197-5p and upregulating NONO expression. These results suggest that IncRNA FAM225A could be explored as a new therapy target in ESCC treatment.
\end{abstract}

Key words: FAM225A; microRNA-197-5p; NONO; ESCC

\section{Introduction}

Esophageal squamous cell carcinoma (ESCC) is the major subclass of esophageal cancer and one of the most life-threatening malignancies with high morbidity and mortality $[1,2]$. In the past decade, the prognosis of ESCC patients have been improved significantly due to the advances in diagnosis technology and therapies such as chemotherapy, radiotherapy and molecular targeted therapy [3,4].
However, the overall 5-year survival rates are still below $20 \%$ in ESCC recurrent patients or ESCC patients with metastasis [5]. Hence, it is crucial to understand the molecular mechanisms of ESCC tumorigenesis and progression, and develop novel therapeutic treatment for ESCC.

Long noncoding RNAs (lncRNAs) are a group of long transcripts (> $200 \mathrm{nt}$ ) without protein translation 
[6]. Accumulating evidence has shown that lncRNAs have important regulatory functions in tumorigenesis and metastasis [7, 8]. Dysregulated expression of lncRNAs has been reported in ESCC $[9,10]$. LncRNAs function as tumor suppressors or oncogenes in the tumorigenesis and metastasis of ESCC [9, 11, 12]. For example, lncRNA HOTTIP was highly expressed in ESCC and promotes metastasis of ESCC via activating of epithelial-mesenchymal transition (EMT) [13]. $\mathrm{CBP} / \mathrm{P} 300$ was reported to enhance the expression of lncRNA linc00460 and promote ESCC carcinogenesis [14]. In the contrast, lncRNA RP11-766N7.4 acts as a tumor suppressor and suppresses EMT process in ESCC [15]. Sun et al. demonstrated that lncRNA CASC2 inhibited ESCC development and progression via increasing the expression of SOCS1 [16].

LncRNA FAM225A is a newly identified lncRNA involved in multiple cancer development and progression $[17,18]$. In nasopharyngeal carcinoma, lncRNA FAM225A functions as a ceRNA to sponge miR-590-3p and miR-1275 expression, and subsequently upregulates ITGB3 expression to promote tumor development and metastasis [17]. FAM225A could also regulate NOTCH3 expression and sponge miR-613 expression to enhance the colorectal cancer development [18]. Nevertheless, the expression pattern and function of lncRNA FAM225A in ESCC has not been studied yet.

In this study, we revealed that IncRNA FAM225A expression was significantly upregulated in ESCC tissues and cell lines and high expression of FAM225A predicted poor prognosis of ESCC patients. Silencing FAM225A suppressed ESCC cell growth and invasive phenotype and inhibited ESCC xenograft development. Moreover, we demonstrated that FAM225A exerted its function via sponging miR-197-5p expression and upregulating the expression of RNA binding protein NONO. Our results suggest that FAM225A/miR-197-5p/NONO axis plays a critical role in ESCC development and progression, which might serve as a promising therapy target for ESCC treatment.

\section{Materials and Methods}

\section{Patient specimens}

ESCC tissues and adjacent normal tissues (118 matched-pairs) were collected from ESCC patients at The Second Affiliated Hospital of Nantong University from 2004 year to 2018 year (NTU cohort). The fresh collected tissues were snap-frozen in liquid nitrogen until further use. All patients provided the written informed consent and the study was reviewed and approved by the Institutional Ethical Review Board of The Second Affiliated Hospital of Nantong
University.

\section{Cell culture}

Four ESCC cell lines (EC9706, KYSE30, KYSE450, and KYSE510) and control cell Het-1A were from Cell Bank, China Academy of Sciences (Shanghai, China). Cells were cultured in DMEM medium supplemented with $10 \%$ fetal bovine serum (Invitrogen, USA) and $1 \%$ antibiotics (Thermo Scientific, USA) at $37^{\circ} \mathrm{C}$ in a $5 \% \mathrm{CO}_{2}$ cell incubator.

\section{Transfection}

The shRNA targeting FAM225A and negative control were purchased from GenePharma company (Shanghai, China). MiR-197-5p mimics, miR-197-5p inhibitor and relative negative control were obtained from RiboBio company (Guangzhou, China). Overexpression plasmids (pcDNA3.1-FAM225A and pcDNA3.1-NONO) and empty vector pcDNA3.1 were purchased from GeneCopoeia company (Guangzhou, China). Transfection was done using Fugene HD reagent (Roche, Switzerland) following the manufacturer's protocol.

\section{Quantitative real-time PCR (qRT-PCR)}

RNA was isolated from tissues samples or cultured cells using Trizol (Thermo Scientific, USA) and reverse-transcribed using Super Transcriptase III (Invitrogen, USA). PowerUP SYBR master mix (Applied Biosystems, USA) was used for qPCR analysis. The relative expression was analyzed by $2^{-\Delta \Delta C T}$ methods. The sequences of primers were listed below: FAM225A, 5'-AAGTCCAGCTGATGCCAG AC-3' and 5'-CACAAGCTTCATCCGAGGGT-3'; miR-197-5p, 5'-CAGTGCAGGGTCCGAGGT-3' and 5'-AACAAGCGGGTAGAGAGGGC-3'; NONO, $5^{\prime}$-CTGATGCGAGAGAGCAGGAG- $3^{\prime}$ and $5^{\prime}$-ATC CGGCATCATAGTGGCAG-3'; GAPDH, 5'-GTCAAG GCTGAGAACGGGAA-3' and 5'-AAATGAGCCCC AGCCTTCTC-3'.

\section{Western blot}

Protein lysate preparation and western blot analysis were performed as described previously [19]. The following primary antibodies were used in the study: NONO (Abcam, ab266244, USA), E-cadherin (Abcam, ab215715, USA), GAPDH (Abcam, ab181602, USA).

\section{Luciferase reporter assay}

Wild type (WT) or mutated (MUT) 3'-UTR of FAM225A or NONO was amplified and constructed into psiCHECK2 luciferase vector (Promega, USA). KYSE510 cells were transfected with luciferase vector, together with miR-197-5p mimics. The relative luciferase activity was analyzed 48 hours post 
transfection using a Dual-luciferase reporter assay kit (Promega, USA).

\section{Cell growth assay}

Cell growth was analyzed by CCK-8 assay using cell counting kit-8 (Sigma-Aldrich, USA) and colony formation assay as previously described [19].

\section{Wound-healing assay}

Transfected cells were cultured in 6-well plates until fluent. The artificial wound was created with a sterile tip and floating cells were washed away with PBS. Cells were cultured for another 48 hours in serum-free medium and cell migration was photographed and calculated at $0 \mathrm{~h}$ and $48 \mathrm{~h}$.

\section{Transwell assay}

Transfected cells $\left(1 \times 10^{5}\right)$ were suspended in 200 $\mu \mathrm{L}$ serum free medium and added to the top layer of a transwell chamber pre-coated with Matrigel (Corning, USA). Bottom chamber was filled with $500 \mu \mathrm{L}$ complete medium with $10 \%$ FBS. The invaded cells were fixed, stained and counted under a light microscope after $24 \mathrm{~h}$ culture.

\section{Immunohistochemical staining}

Tissue sections from different groups were deparaffinized, rehydrated and blocked with $1 \%$ FBS. Then, tissue sections were stained with anti-Ki-67 antibodies (Abcam, ab270650) overnight at $4^{\circ} \mathrm{C}$. The staining was visualized using the DAKO EnVision System (DAKO, Denmark).

\section{Xenograft tumor model}

All animal experiments were reviewed and approved by the Institutional Animal Care and Use Committee of The Second Affiliated Hospital of Nantong University. Xenograft tumor model was established by inoculating KYSE510 cells $\left(5 \times 10^{6}\right)$ stably transfected with sh-FAM225A or negative control into nude mice (male, 5 weeks old). Tumor growth was measured every week. On week 5, mice were euthanized and xenograft tumors were dissected and weighted. The tumor tissue sections were fixed and paraffin-embedded for hematoxylin and eosin (H\&E) and Ki-67 staining analysis.

\section{In situ hybridization}

In Situ Hybridization (ISH) was conducted using an In situ hybridization kit (Boster, China). Tissue sections were processed to de-paraffinization, dehydration and digested with pepsin. Subsequently, sections were incubated with FAM225A-specific probe at $50^{\circ} \mathrm{C}$ overnight. Sections were further incubated with biotinylated anti-Digoxin (Boster, China), stained with biotinylated peroxidase, and visualized with 3,3'-diaminobenzidine substrate.

\section{Statistical analysis}

The results of at least three experiments were shown as mean \pm standard deviation (SD). Kaplan-Meier analysis was performed to analyze the overall survival (OS) and disease-free survival (DFS) of ESCC patients with high or low FAM225A expression. The statistical analysis was conducted using GraphPad Prism (V6, Prism, USA) and a P value $<0.05$ was considered statistical significance.

\section{Results}

\section{LncRNA FAM225A is highly expressed in ESCC and predicts poor prognosis}

To investigate the lncRNA FAM225A expression in ESCC, we performed qPCR analysis in 30-paired ESCC tissues and adjacent tissues. LncRNA FAM225A expression was much higher in ESCC tissues than that in normal tissues (Figure 1A). Microarray analysis using 30-paired ESCC tissues and adjacent tissues also confirmed high expression of FAM225A in ESCC tissues (Figure 1B). In addition, lncRNA FAM225A expression was markedly higher in ESCC cell lines (EC9706, KYSE30, KYSE450, and KYSE510) than in control Het-1A cells (Figure 1C). In situ hybridization (ISH) was performed using ESCC tissue microarray and the relative expression of lncRNA FAM225A was scored as 1 to 5 according to the staining intensity. The ISH results suggested that ESCC tissues had significantly upregulated lncRNA FAM225A expression than that in adjacent tissues (Figure 1D). Furthermore, Kaplan-Meier analysis showed that ESCC patients with higher lncRNA FAM225A expression had no statistic difference for overall survival (OS) (Figure 1E), while there was significant worse disease-free survival (DFS) in comparison with patients had low lncRNA FAM225A expression (Figure 1F).

To further validate the expression of lncRNA FAM225A in ESCC, RNA sequencing data of ESCC from the Cancer Genome Atlas (TCGA) was analyzed and ESCC tissues had significantly higher lncRNA FAM225A expression (Figure 2A). Intriguingly, we found that ESCC tissues with advanced TNM stages had higher lncRNA FAM225A expression levels than those in ESCC tissues of TNM stage I (Figure 2B). Consistently, ESCC patients with high lncRNA FAM225A level had worse DFS, but not OS, compared with patients with low lncRNA FAM225A levels in ESCC TCGA cohort (Figure $2 C$ and D). Our results suggested that lncRNA FAM225A expression was overexpressed in ESCC tissues and predicted unfavorable prognosis of ESCC patients. 
A

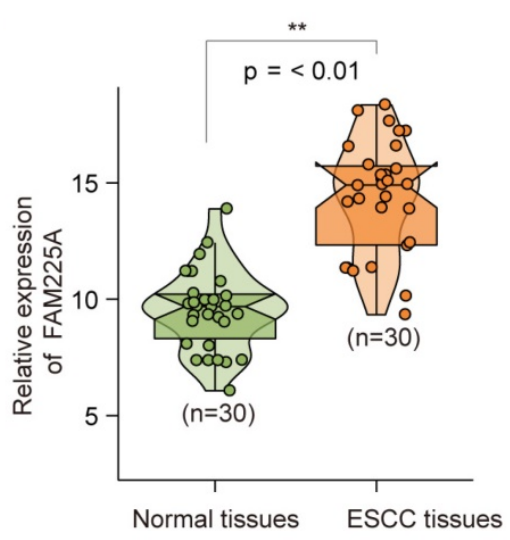

B

ESCC Patient ID

123456789101112131415161718192021222324252627282930

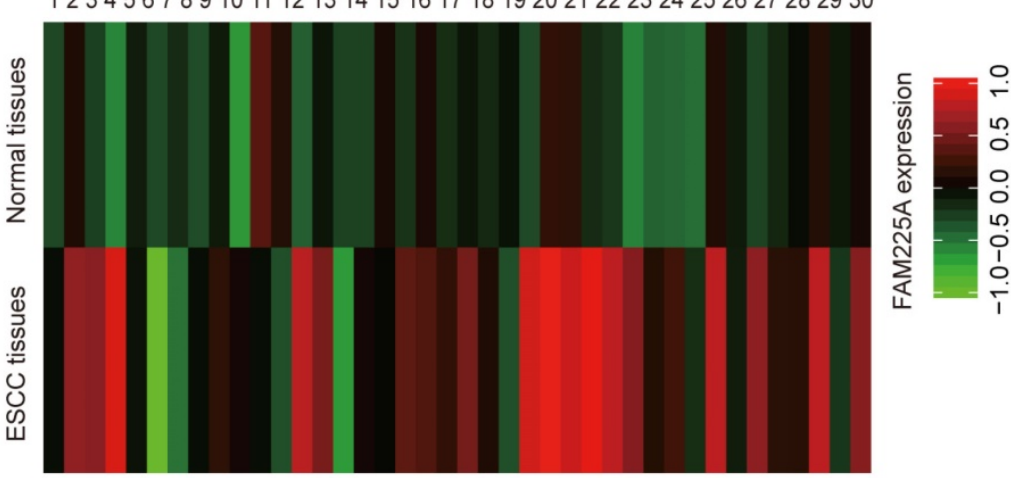

C

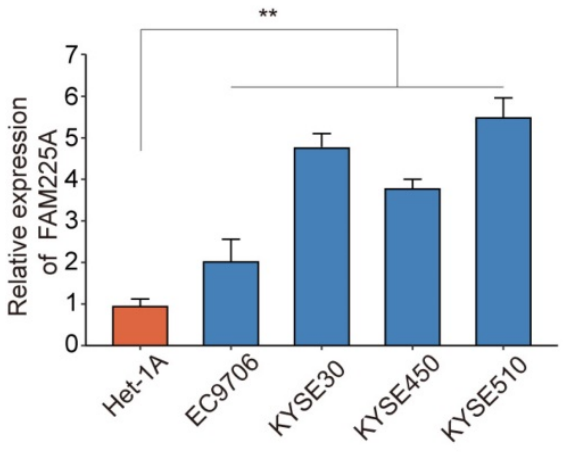

D

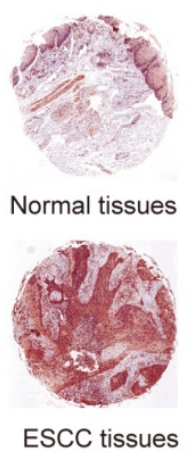

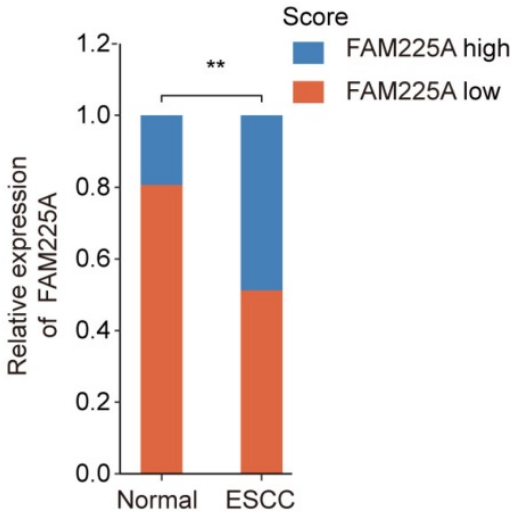

$E$

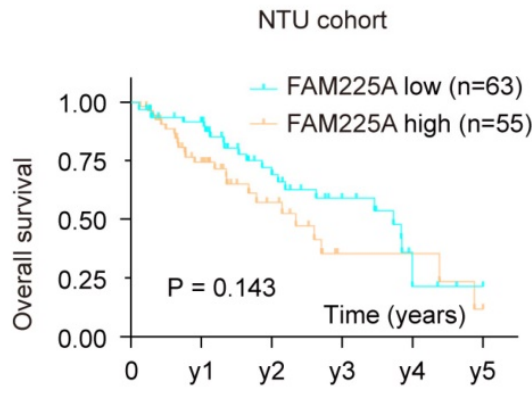

$\mathrm{F}$

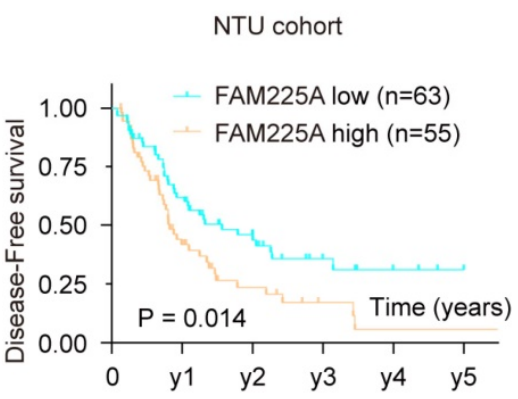

Figure 1. LncRNA FAM225A is upregulated in ESCC and predicts poor prognosis. (A) The expression of IncRNA FAM225A in 30-paired ESCC tissues and adjacent normal tissues was analyzed by qRT-PCR. (B) Heat map illustrated the microarray analysis of relative expression of IncRNA FAM225A in ESCC tissues and adjacent normal tissues from 30 ESCC patients. (C) The expression of IncRNA FAM225A in ESCC cell lines (EC9706, KYSE30, KYSE450, and KYSE510) and control cell line Het-1 A was examined by qRT-PCR. (D) In situ hybridization (ISH) was performed to examine the expression of IncRNA FAM225A in ESCC tissues and the relative expression of IncRNA FAM225A was scored as 1 to 5 based on the staining intensity. Relative ISH staining of IncRNA FAM225A in normal tissues and ESCC tissues and the distribution of different scores of FAM225A ISH staining intensity were shown. (E) Kaplan-Meier analysis of overall survival (OS) in ESCC patients with high or low IncRNA FAM225A expression was performed. (F) Kaplan-Meier analysis of Disease-free survival (DFS) in ESCC patients with high or low IncRNA FAM225A expression was performed. ** $\mathrm{P}<0.01$.

\section{Knockdown of IncRNA FAM225A suppresses ESCC cell proliferation, migration and invasion}

To study the function of lncRNA FAM225A on ESCC cell proliferation and metastasis ability, two ESCC cell lines (KYSE30 and KYSE510) with highest levels of lncRNA FAM225A, were transfected with shRNA targeting lncRNA FAM225A to knockdown its expression (Figure 3A). Functional assays showed that knockdown of lncRNA FAM225A significantly dampened cell proliferation and colony formation of ESCC cells (Figure 3B and 3C). Furthermore, wound-healing assay and transwell assay demonstrated that suppression of FAM225A dampened the migration and invasion capabilities of ESCC cells (Figure 3D-3F). These findings indicated that lncRNA FAM225A functioned as an oncogene and promoted ESCC cell proliferation, migration and invasion. 
A

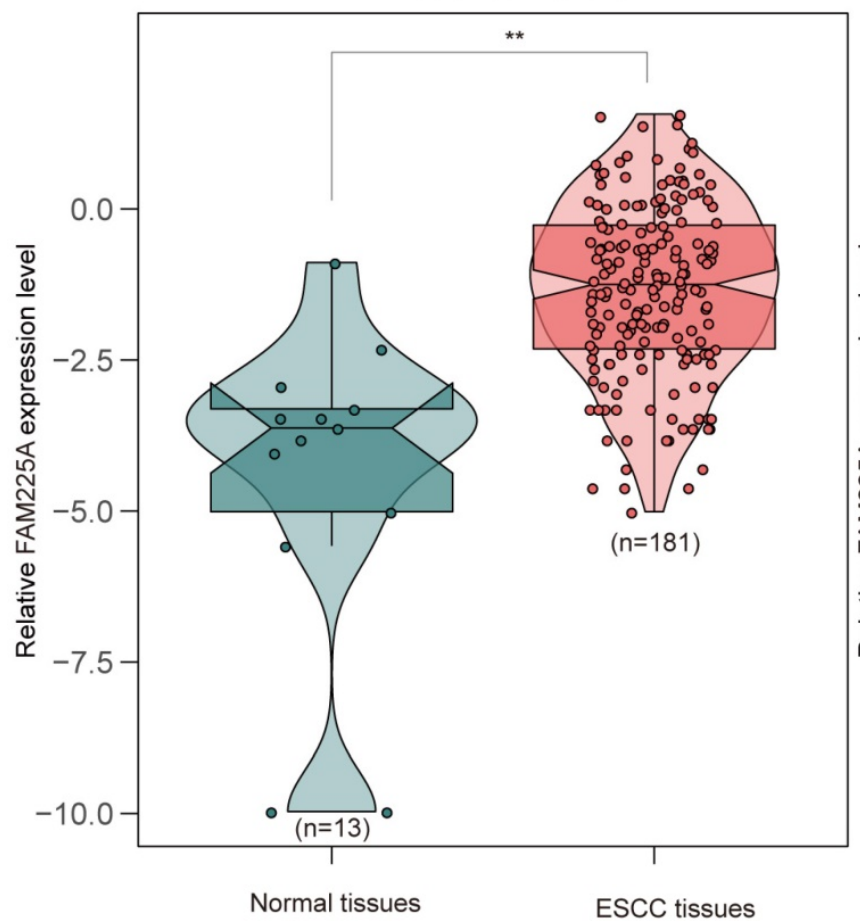

C

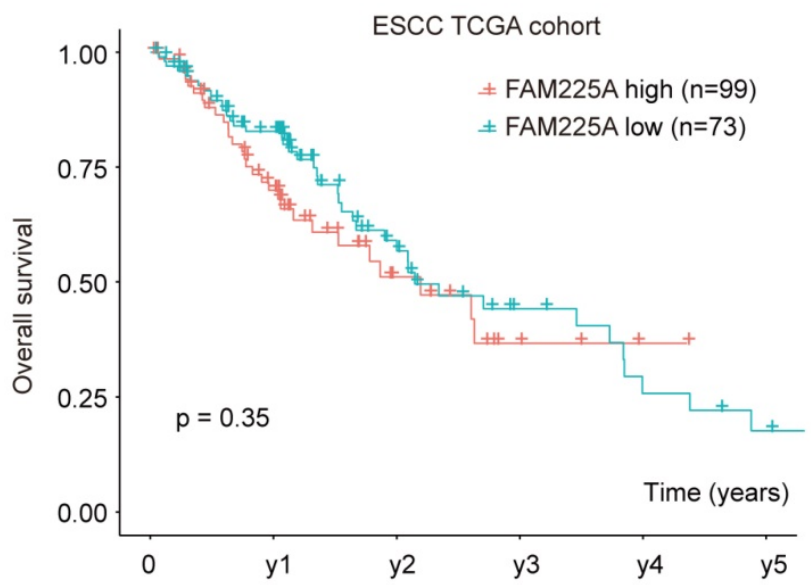

B

ESCC TCGA cohort

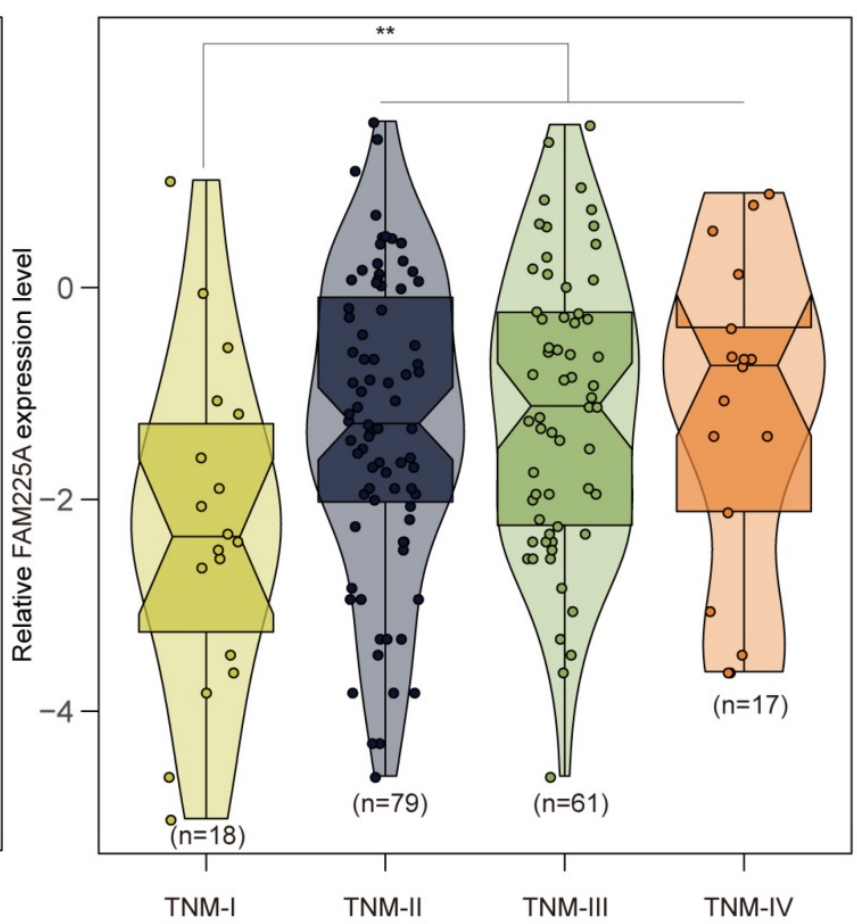

D

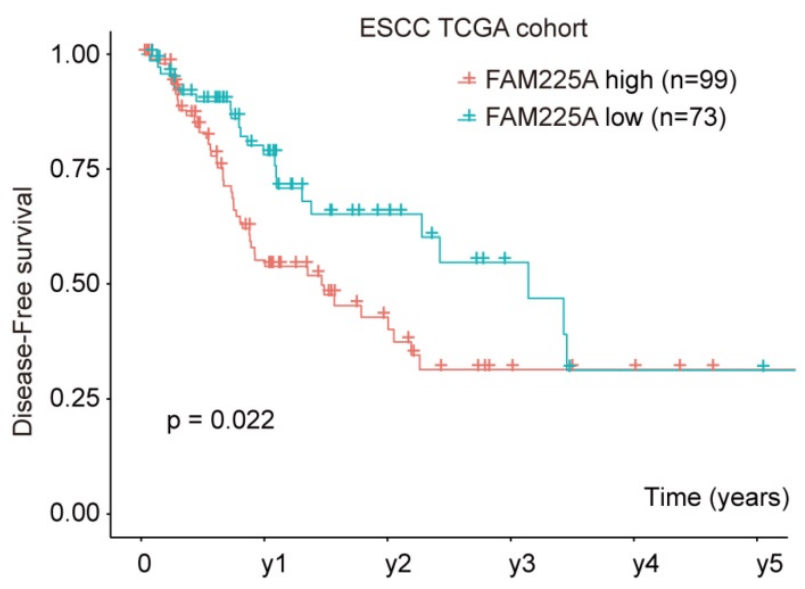

Figure 2. ESCC TCGA datasets analysis indicates high expression of IncRNA FAM225A correlates poor outcome in ESCC patients. (A) RNA sequencing data of ESCC from the Cancer Genome Atlas (TCGA) was analyzed and the relative expression of IncRNA FAM225A in normal tissues and ESCC tissues was shown. (B) The relative expression of IncRNA FAM225A in ESCC tissues with different TNM stages was analyzed in ESCC TCGA cohort. (C) Kaplan-Meier analysis of OS in ESCC patients with high or low IncRNA FAM225A expression in ESCC TCGA cohort was performed. (D) Kaplan-Meier analysis of DFS in ESCC patients with high or low IncRNA FAM225A expression in ESCC TCGA cohort was performed. ** $\mathrm{P}<0.01$.

\section{Knockdown of IncRNA FAM225A inhibits ESCC tumorigenesis in vivo}

We also evaluated the function of lncRNA FAM225A on ESCC tumorigenesis in vivo. Xenograft tumor model of ESCC was established by transplanting stable transfected KYSE510 cells (Mock or sh-FAM225A) into nude mice. As shown in Figure 4A-4C, knockdown of lncRNA FAM225A significantly inhibited ESCC tumor growth, with markedly delayed tumor development and decreased tumor weights in sh-FAM225A group. In addition, immunohistochemical staining suggested that knockdown of FAM225A markedly decreased the expression of proliferation marker Ki-67 in xenograft tumors. H\&E counter-staining was performed to visualize the tumor tissues (Figure 4D). Our data indicated that knockdown of FAM225A suppressed ESCC tumorigenesis in vivo. 
A

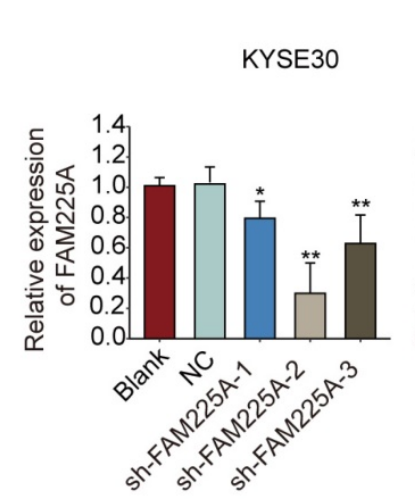

C

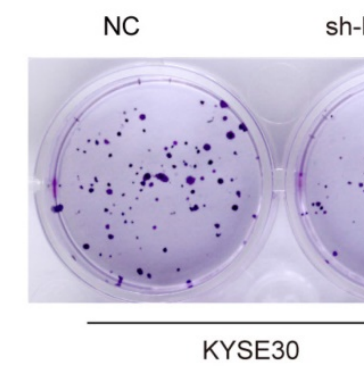

sh-FAM225A-2

NC
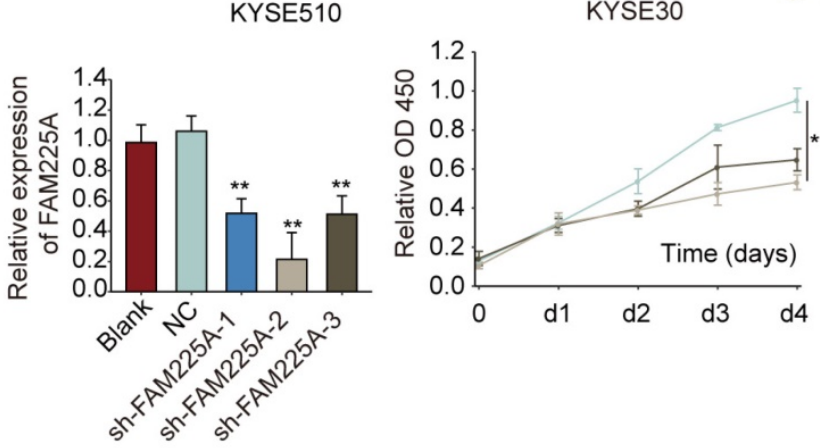

- NC

- sh-FAM225A-1

sh-FAM225A-2

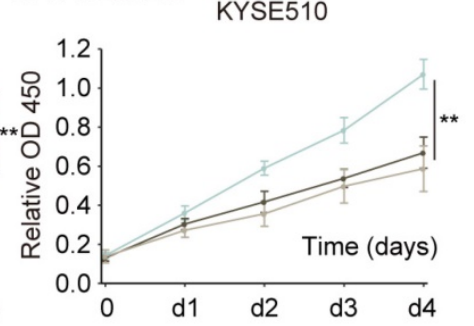

E

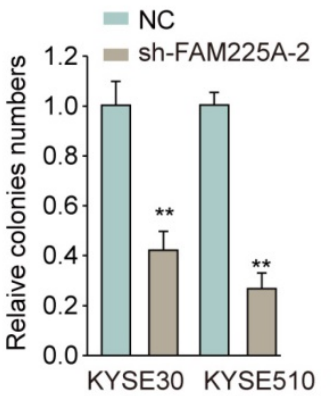

D
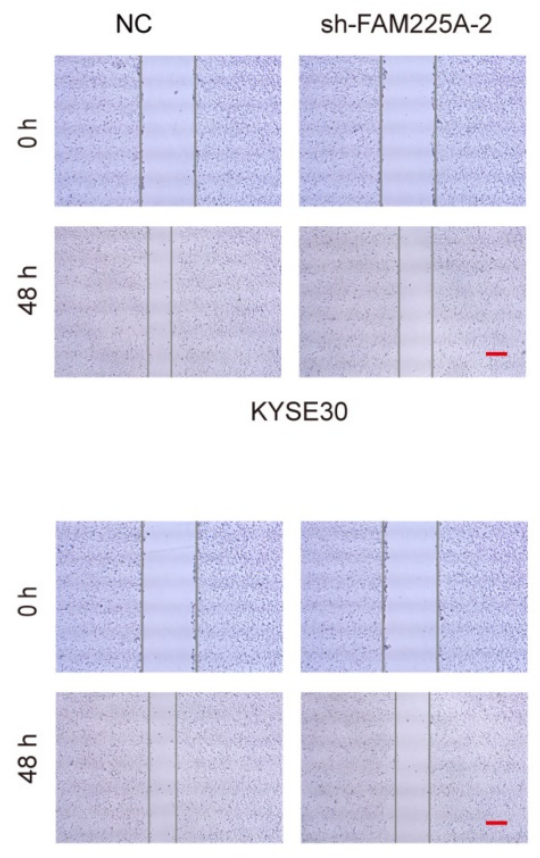

KYSE510
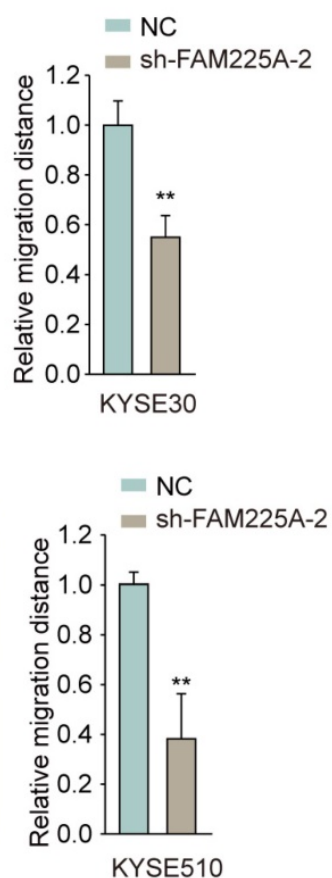

Figure 3. Knockdown of IncRNA FAM225A suppresses ESCC cell proliferation, migration and invasion. (A) KYSE30 or KYSE510 cells were transfected with negative control (NC), sh-FAM225A-1/2/3, or left untreated (blank). The relative expression of FAM225A was examined by qRT-PCR 48 hours later. (B-F) KYSE30 or KYSE510 cells were transfected with NC, sh-FAM225A-1, or sh-FAM225A-2. (B) Cell proliferation was analyzed by CCK-8 assay at indicated time. (C) Cell growth was analyzed by colony formation. (D) Cell migration was analyzed by wound-healing assay. (E) Cell invasion was analyzed by transwell assay. $* P<0.05, * * P<0.01$.

\section{LncRNA FAM225A functions as a ceRNA via sponging miR-197-5p}

To study how IncRNA FAM225A exerts its regulatory function on ESCC, we utilized the online tool StarBase to predict the potential miRNAs interacting with FAM225A. The top five predicted miRNAs with highest score were further evaluated by transfection of sh-FAM225A in KYSE30 cells (Figure 5A). LncRNA FAM225A knockdown significantly 
enhanced miR-197-5p expression and FAM225A had the complementary binding sequences against miR-197-5p (Figure 5B). Intriguingly, the miR-197-5p expression was negatively associated with the expression of lncRNA FAM225A in ESCC tissues (Figure 5C). Quantitative RT-PCR analysis showed that miR-197-5p expression was markedly lower in ESCC tissues and cell lines than that in non-tumor tissues and control cell line (Figure 5D and 5E). In addition, we revealed that overexpression of lncRNA FAM225A suppressed miR-197-5p expression while miR-197-5p mimics reversed the inhibitory effect of lncRNA FAM225A in KYSE30 or KYSE510 cells (Figure 5F and 5G). To further validate the relationship between lncRNA FAM225A and miR-197-5p, we performed the luciferase reporter assay and the results revealed that miR-197-5p mimics specifically inhibited the luciferase activity of WT
3'-UTR of lncRNA FAM225A (Figure 5H). These results indicated that lncRNA FAM225A sponged miR-197-5p expression in ESCC.

\section{LncRNA FAM225A exerts its regulatory function on ESCC cell proliferation and metastasis via modulating miR-197-5p expression}

To further study the interaction between lncRNA FAM225A and miR-197-5p, KYSE30 or KYSE510 cells were co-transfected with miR-197-5p mimics or mock control, with or without pcDNA3.1-FAM225A. Cell growth assays demonstrated that overexpression of lncRNA FMA225A enhanced cell growth and miR-197-5p mimics suppressed ESCC cell proliferation (Figure 6A and 6B). However, MiR-197-5p overexpression antagonized the promotion effect of pcDNA3.1-FAM225A (Figure 6A
A

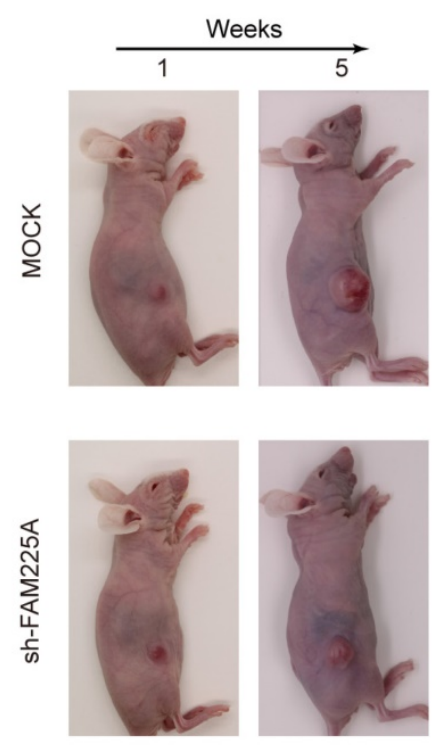

C

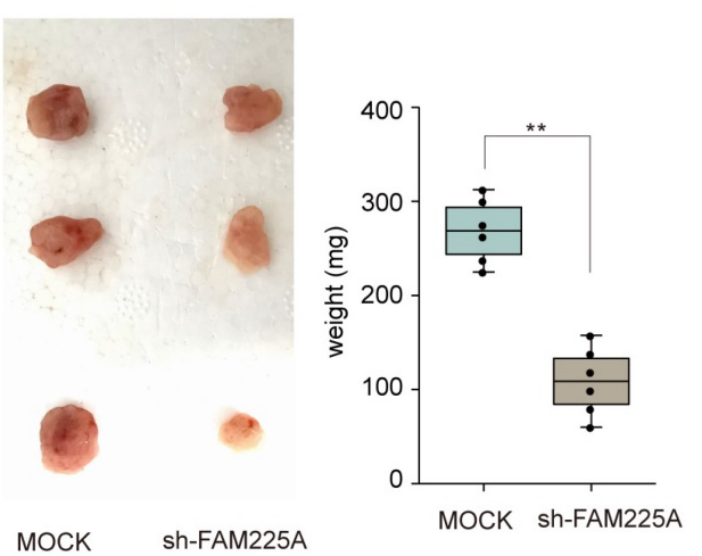

B

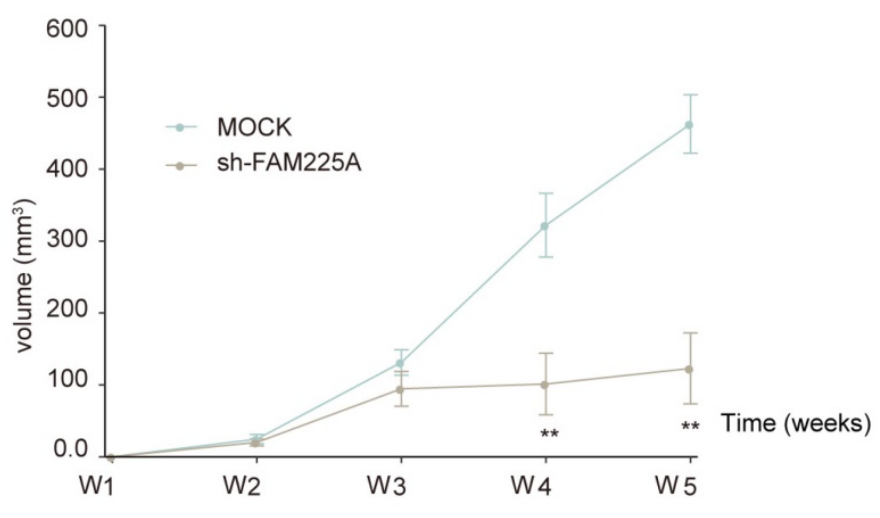

D

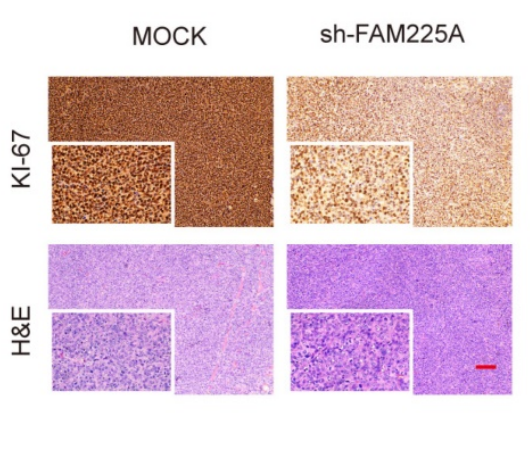

Figure 4. Knockdown of IncRNA FAM225A inhibits ESCC xenograft tumor development in vivo. KYSE510 cells stably transfected with sh-FAM225A or mock control were transplanted into the right flank of nude mice to establish ESCC xenograft tumor model. (A) The representative images of nude mice in different groups at week 1 and weeks 5 were shown. (B) Tumor growth was monitored at indicated time points. (C) Tumor weights from different groups were analyzed. (D) The representative immunohistochemical staining of $\mathrm{Ki}-67$ and $\mathrm{H} \& \mathrm{E}$ staining of xenograft tumor sections and the quantification of Ki-67 expression were analyzed. $* * \mathrm{P}<0.01$. 
and 6B). Further, we found that FAM225A promoted ESCC invasion and overexpression of miR-197-5p dampened cell invasion of KYSE30 or KYSE510 cells, whereas overexpression FAM225A together with miR-197-5p showed the similar invasive ability of
ESCC cells with control group (Figure 6C). Thus, we concluded that IncRNA FAM225A exerted its regulatory effect on ESCC cell proliferation and metastasis via downregulating miR-197-5p expression.

A

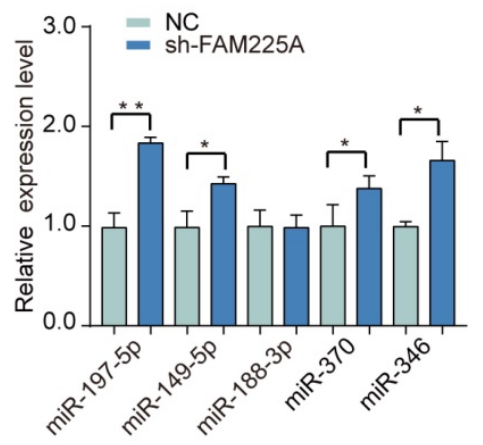

FAM225A expression

$\mathrm{F}$

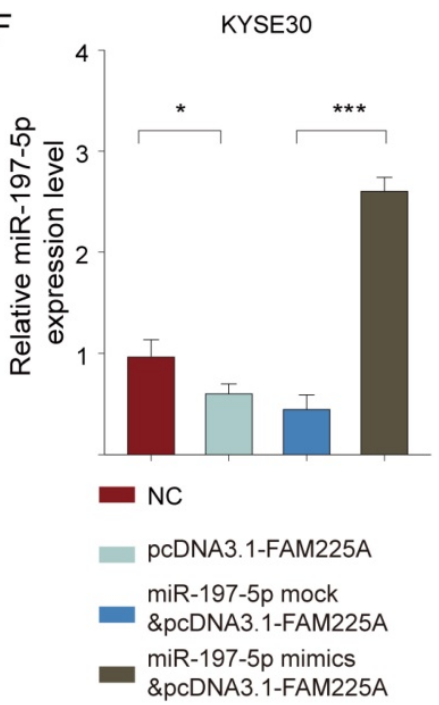

B

\author{
Wt FAM225A 5'AGAGCUGUGGACAGUCAUGAAGGG 3' \\ I| | । I | | | | | | | \\ hsa-miR-197-5p 3'GUCUGAGGCCUGACCUUACUUCCU 5' \\ Mut FAM225A 5'AGAGUGGUAAACAUUCGCUCCUUG 3'
}

C

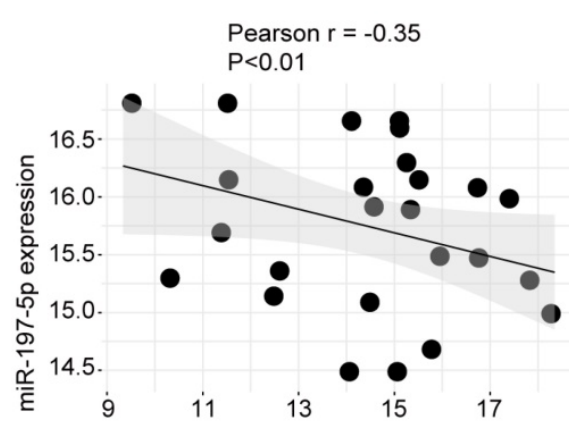

D

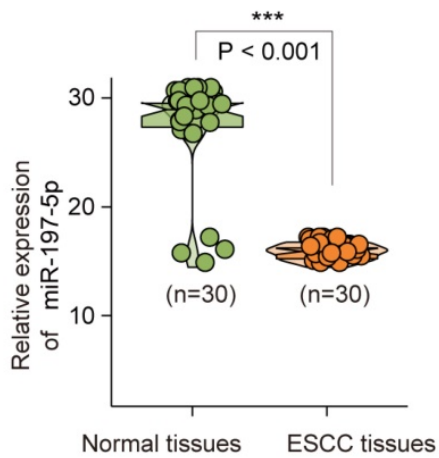

E

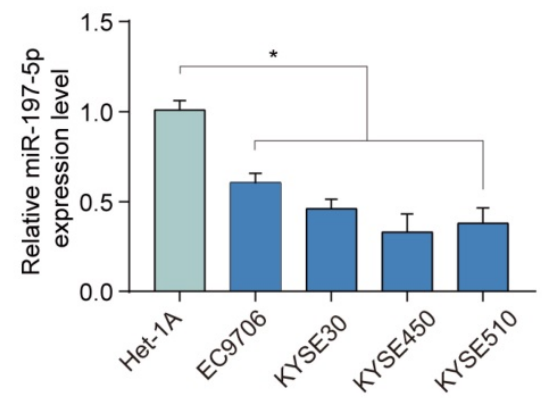

Figure 5. LncRNA FAM225A functions as a ceRNA via sponging miR-197-5p. (A) KYSE30 cells were transfected with negative control (NC) or sh-FAM225A. The expression levels of miR-197-5p, miR-149-5p, miR-188-3p, miR-370, and miR-346 were analyzed by qPCR. (B) The putative binding sequences between IncRNA FAM225A and miR-197-5p was shown. (C) Pearson analysis of the correlation between IncRNA FAM225A expression and miR-197-5p expression. (D) The relative expression of miR-197-5p was analyzed in ESCC tissues and non-tumor control tissues by qRT-PCR. (E) The relative expression of miR-197-5p was analyzed in ESCC cell lines and control Het-1A cells by qRT-PCR. (F, G) KYSE30 or KYSE510 cells were transfected with NC, pcDNA3.1-FAM225A, miR-197-5p mock \& pcDNA3.1-FAM225A, or miR-197-5p mimics \& pcDNA3.1-FAM225A. The relative expression of miR-197-5p was analyzed by qRT-PCR 48 hours post transfection. (H) KYSE510 cells were co-transfected with luciferase reporter vector containing WT or mutated 3'-UTR of IncRNA FAM225A, with or without miR-197-5p mimics. The relative luciferase activity was analyzed 48 hours later by using a dual-Glo luciferase assay kit. $* \mathrm{P}<0.05, * * \mathrm{P}<0.01$. 
A
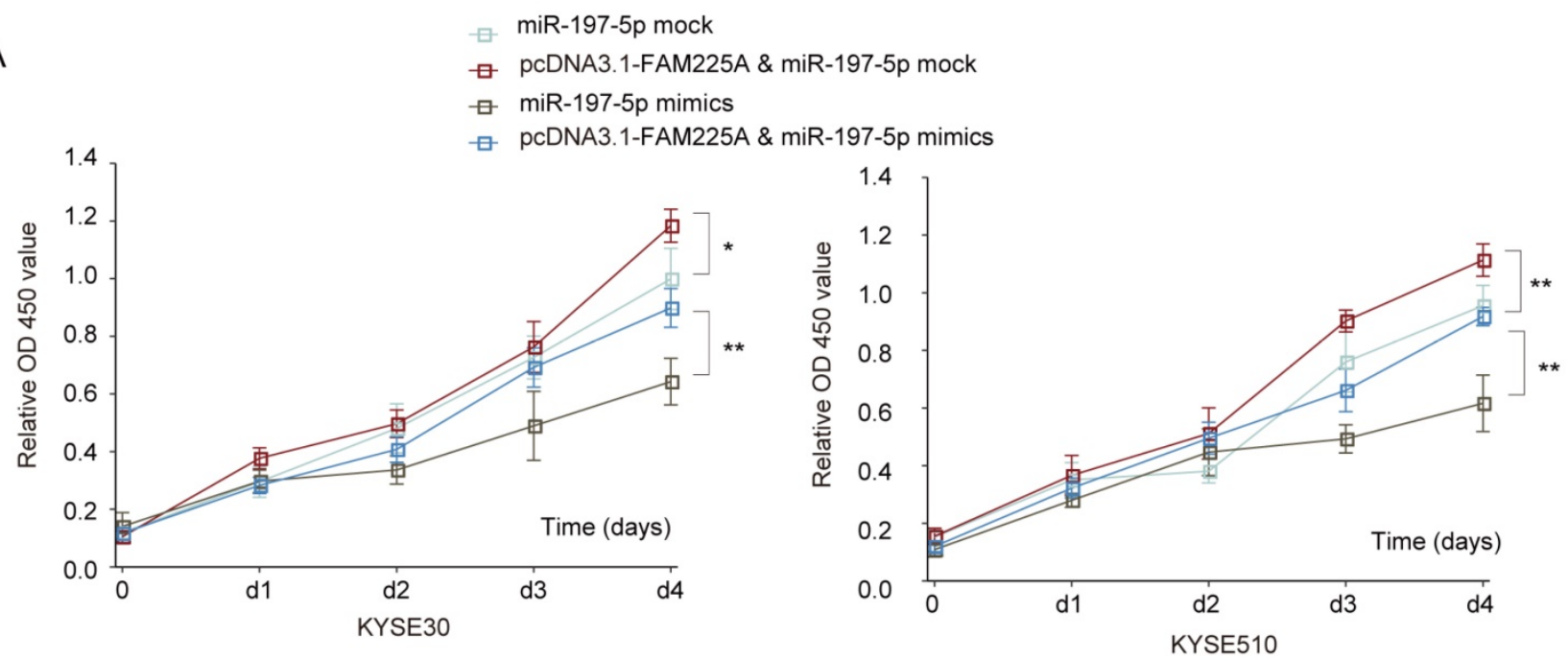

B
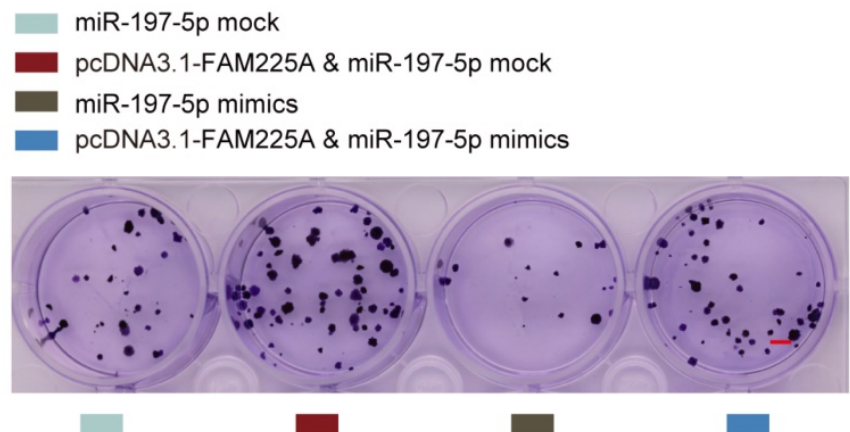

KYSE30

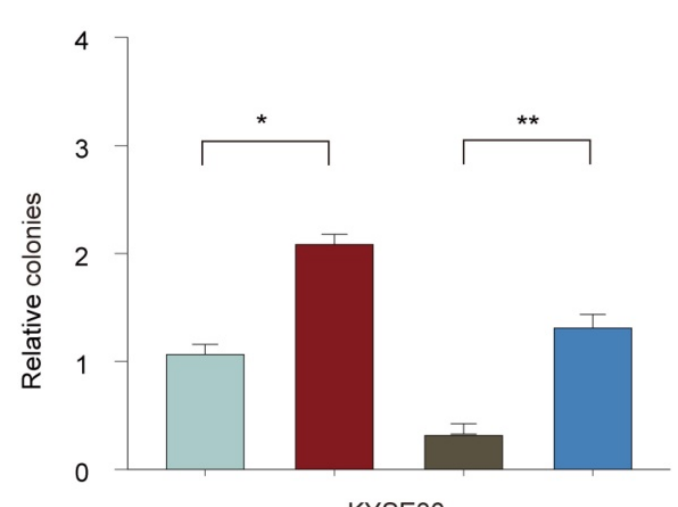

C

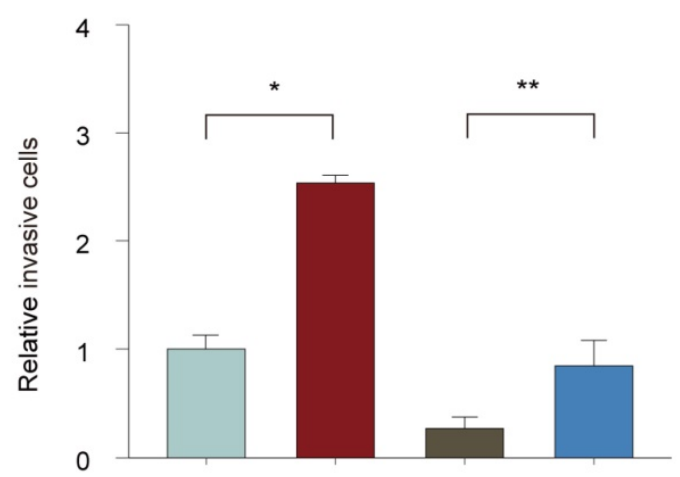

KYSE30

KYSE30

Figure 6. LnCRNA FAM225A exerts its regulatory function on ESCC cell proliferation and metastasis via downregulating miR-197-5p expression. KYSE30 or KYSE5 10 cells were transfected with miR-197-5p mock control, pCDNA3.1-FAM225A \& miR-197-5p mock, miR-197-5p mimics, or pcDNA3.1-FAM225A \& miR-197-5p mimics. (A) Cell proliferation was analyzed by CCK-8 assay. (B) Cell growth was evaluated by colony formation assay. (C) Cell invasion was evaluated by transwell assay. $*$ P $<$ $0.05, * * \mathrm{P}<0.01$

\section{MiR-197-5p negatively regulates NONO expression and TGF- $\beta$ signaling in ESCC cells}

To find out the target gene regulated by miR-197-5p, we searched the potential miR-197-5p targets using TargetScan and miRDB online tools.
RNA-binding protein NONO was predicted as the top candidate and had the complementary binding sequences of miR-197-5p (Figure 7A). Luciferase reporter assay further confirmed the interaction between miR-197-5p and NONO (Figure 7B). In addition, we found that miR-197-5p overexpression 
suppressed NONO expression whereas miR-197-5p inhibition enhanced NONO expression in ESCC cells (Figure 7C). We also performed Gene Set enrichment Analysis (GSEA) to investigate the potential signaling pathways involved in miR-197-5p/NONO regulation of ESCC. As shown in Figure 7D, TGF- $\beta$ signaling signature genes were enriched in NONO high expression group. Moreover, we confirmed that in
KYSE30 or KYSE510 cells, overexpression of miR-197-5p inhibited MONO and TGF- $\beta$ expression and overexpression of MONO restored MONO and TGF- $\beta$ levels in ESCC cells transfected with miR-197-5p (Figure 7E). These data suggested that miR-197-5p negatively regulated NONO expression and TGF- $\beta$ signaling in ESCC cells.
A
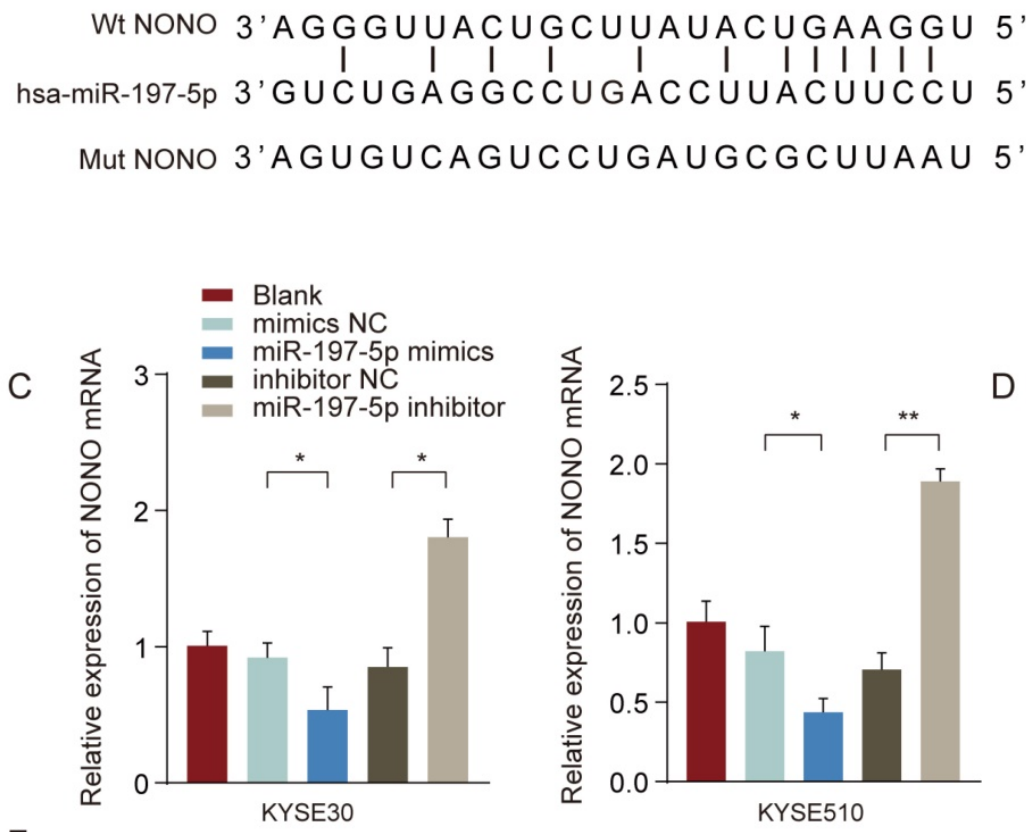

$\mathrm{E}$

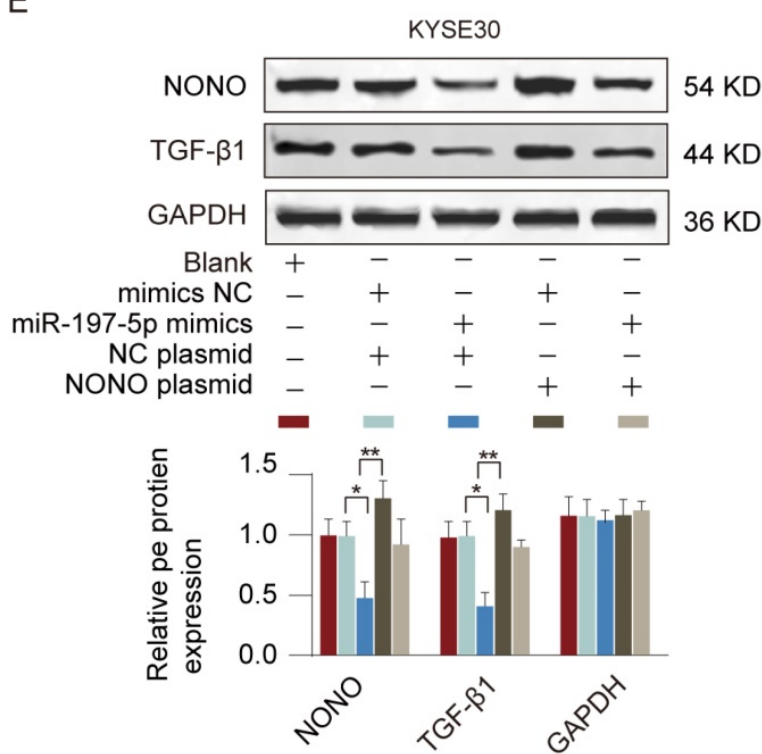

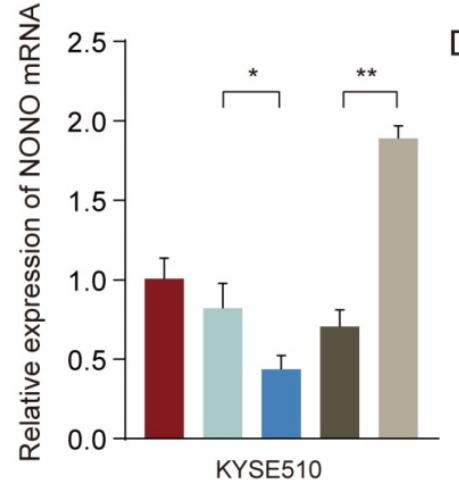

D

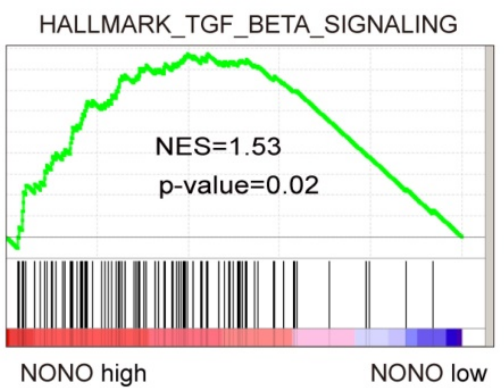

KYSE510
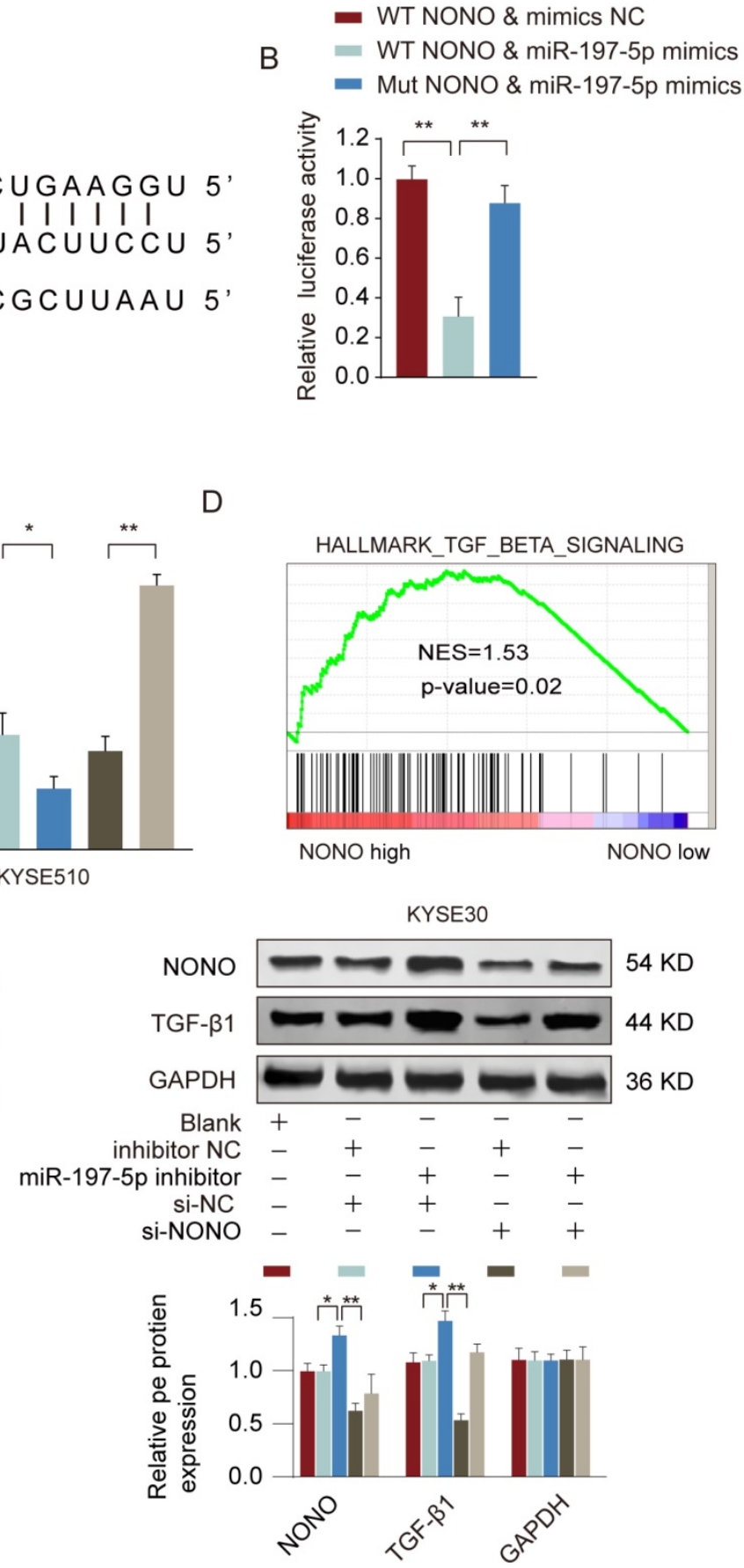

Figure 7. MiR-197-5p negatively regulates NONO expression and TGF- $\boldsymbol{\beta}$ signaling in ESCC cells. (A) The predicted complementary binding sequences between miR-197-5p and NONO was shown. (B) KYSE510 cells were transfected with reporter vector containing WT or mutated 3'-UTR of NONO, together with miR-197-5p mimics or negative control. The relative luciferase activity was analyzed 48 hours post transfection. (C) KYSE30 or KYSE510 cells were transfected with miR-197-5p mimics, miR-197-5p inhibitor or relative negative controls. The relative expression of NONO was analyzed 48 hours later. (D) GSEA analysis revealed TGF-beat signaling signature genes were enriched in NONO high group. (E) KYSE30 or KYSE5 10 cells were transfected with miR-197-5p mimics or negative control, with or without NONO overexpression plasmid. The protein expression levels of NONO and TGF- $\beta$ were examined by western blot. $* P<0.05, * * P<0.01$. 


\section{Discussion}

LncRNAs have been demonstrated to play crucial roles in tumorigenesis and metastasis of ESCC [8]. LncRNAs could be utilized as early diagnostic biomarkers or prognosis predictors [20, 21]. They exert regulatory function via binding to miRNA expression or transcription factors as decoys or acting as dynamic scaffold to transiently assemble with cofactors [22]. In this study, we identified lncRNA FAM225A functioned as a ceRNA via sponging miR-197-5p and modulating the expression of NONO to promote the ESCC development and progression. Knockdown of FAM225A inhibited ESCC proliferation and metastasis. These findings demonstrate that FAM225A is an oncogenic lncRNA in ESCC, which could be utilized as therapeutic target for ESCC treatment.

LncRNA FAM225A was identified via microarray screening to be one of the most upregulated lncRNA in nasopharyngeal carcinogenesis. FAM225A enhanced ITGB3 expression via sponging miR-590-3p and miR-1275 [17]. In another study, FAM225A was overexpressed in colorectal cancer tissues and cell lines and predicted unfavorable outcome of colorectal cancer patients [18]. Similar to the published results, we also demonstrated that lncRNA FAM225A was highly expressed in ESCC and functioned as an oncogenic lncRNA. However, we validated that in ESCC, FAM225A interacted with miR-197-5p and the expression of FAM225A was significantly negative associated with miR-197-5p expression in ESCC tissues.

MiR-197-5p was demonstrated to act as circulating biomarker for heart failure [23]. It has been reported that in carcinogenesis of fibrosarcoma, miR-197-5p exerts its regulatory role through inhibiting of KIAA0101 [24]. MiR-197-5p Overexpression markedly suppressed fibrosarcoma cell proliferation and promoted cell apoptosis [24]. In glioma, miR-197-5p interacted with lncRNA DLX6-AS1 and suppressed transcription factor E2F1 expression, thus dampened glioma development and progression [25]. We revealed the function of miR-197-5p as a tumor suppressor in ESCC. MiR-197-5p expression was downregulated in ESCC tissues and cell lines. Overexpression of miR-197-5p antagonized the oncogenic function of FAM225A in ESCC cells.

The RNA binding protein NONO has been demonstrated to participate in various biological processes including tumorigenesis and metastasis [26]. Upregulation of NONO expression is correlated with unfavorable prognosis with multiple tumors, such as bladder cancers, prostate cancers, and malignant melanoma [27-29]. Rui Cheng et al. showed that NONO expression was dramatically upregulated in ESCC and knockdown of NONO using siRNA inhibited ESCC cell proliferation and promoted ESCC apoptosis [30]. However, in that study, how NONO expression was regulated was not clear. Consistently, we found that NONO was upregulated in ESCC and it was a direct target of miR-197-5p. Moreover, we demonstrated that miR-197-5 $p$ negatively regulated NONO expression and TGF- $\beta 1$ signaling. Nevertheless, the contribution of TGF- $\beta 1$ signaling to ESCC development and progression needs further investigation.

In conclusion, we explored the expression and function effects of lncRNA FAM225A, as well as the underlying regulatory mechanisms in ESCC development and progression. Our findings indicate that lncRNA FAM225A promotes ESCC tumorigenesis and metastasis via sponging miR-197-5p and upregulating NONO expression. The regulatory axis of FAM225A/miR-197-5p/NONO might be utilized to develop novel therapy for ESCC patients.

\section{Abbreviations}

ESCC: Esophageal squamous cell carcinoma; TMA: Tissue microarray; RT-qPCR: Real-time reverse transcription polymerase chain reaction; IHC: Immunohistochemical; ISH: In Situ Hybridization; OS: Overall survival; DFS: Disease-free survival; TCGA: Cancer Genome Atlas.

\section{Acknowledgements}

This work was supported by Project of Shanghai Science and Technology Commission (Nos. 18441902300), National Natural Science Foundation of China (Nos. 81974053), China postdoctoral science foundation (Nos. 2018M632352) and Jiangsu Youth Medical Key Talents (No. QNRC2016405).

\section{Competing Interests}

The authors have declared that no competing interest exists.

\section{References}

1. Liang H, Fan JH, Qiao YL. Epidemiology, etiology, and prevention of esophageal squamous cell carcinoma in China. Cancer Biol Med. 2017; 14: 33-41.

2. Abbas G, Krasna M. Overview of esophageal cancer. Ann Cardiothorac Surg. 2017; 6: 131-6.

3. Liu Y, Xiong Z, Beasley A, D'Amico T, Chen XL. Personalized and targeted therapy of esophageal squamous cell carcinoma: an update. Ann N Y Acad Sci. 2016; 1381: 66-73

4. Ohashi S, Miyamoto S, Kikuchi O, Goto T, Amanuma Y, Muto M. Recent Advances From Basic and Clinical Studies of Esophageal Squamous Cell Carcinoma. Gastroenterology. 2015; 149: 1700-15.

5. Pakzad R, Mohammadian-Hafshejani A, Khosravi B, Soltani S, Pakzad I, Mohammadian M, et al. The incidence and mortality of esophageal cancer and their relationship to development in Asia. Ann Transl Med. 2016; 4: 29. 
6. Li Y, Wang X. Role of long noncoding RNAs in malignant disease (Review). Mol Med Rep. 2016; 13: 1463-9.

7. Serghiou S, Kyriakopoulou A, Ioannidis JP. Long noncoding RNAs as novel predictors of survival in human cancer: a systematic review and meta-analysis. Mol Cancer. 2016; 15: 50.

8. Talebi A, Masoodi M, Mirzaei A, Mehrad-Majd H, Azizpour M, Akbari A. Biological and clinical relevance of metastasis-associated long noncoding RNAs in esophageal squamous cell carcinoma: A systematic review. J Cell Physiol. 2020; 235: 848-68.

9. Cao W, Wu W, Shi F, Chen X, Wu L, Yang K, et al. Integrated analysis of long noncoding RNA and coding RNA expression in esophageal squamous cell carcinoma. Int J Genomics. 2013; 2013: 480534.

10. Ma J, Xiao Y, Tian B, Chen S, Zhang B, Wu J, et al. Genome-wide analyses of long non-coding RNA expression profiles and functional network analysis in esophageal squamous cell carcinoma. Sci Rep. 2019; 9: 9162.

11. Hao Y, Wu W, Shi F, Dalmolin RJ, Yan M, Tian F, et al. Prediction of long noncoding RNA functions with co-expression network in esophageal squamous cell carcinoma. BMC Cancer. 2015; 15: 168.

12. Wei G, Luo H, Sun Y, Li J, Tian L, Liu W, et al. Transcriptome profiling of esophageal squamous cell carcinoma reveals a long noncoding RNA acting as a tumor suppressor. Oncotarget. 2015; 6: 17065-80.

13. Chen X, Han H, Li Y, Zhang Q, Mo K, Chen S. Upregulation of long noncoding RNA HOTTIP promotes metastasis of esophageal squamous cell carcinoma via induction of EMT. Oncotarget. 2016; 7: 84480-5.

14. Liang $\mathrm{Y}, \mathrm{Wu} \mathrm{Y}$, Chen $\mathrm{X}$, Zhang S, Wang $\mathrm{K}$, Guan $\mathrm{X}$, et al. A novel long noncoding RNA linc00460 up-regulated by CBP/P300 promotes carcinogenesis in esophageal squamous cell carcinoma. Biosci Rep. 2017; 37.

15. Yao GL, Pan CF, Xu H, Wei K, Liu B, Zhai R, et al. Long noncoding RNA RP11-766N7.4 functions as a tumor suppressor by regulating epithelial-mesenchymal transition in esophageal squamous cell carcinoma. Biomed Pharmacother. 2017; 88: 778-85.

16. Sun K, Zhang G. Long noncoding RNA CASC2 suppresses esophageal squamous cell carcinoma progression by increasing SOCS1 expression. Cell Biosci. 2019; 9: 90

17. Zheng ZQ, Li ZX, Zhou GQ, Lin L, Zhang LL, Lv JW, et al. Long Noncoding RNA FAM225A Promotes Nasopharyngeal Carcinoma Tumorigenesis and Metastasis by Acting as ceRNA to Sponge miR-590-3p/miR-1275 and Upregulate ITGB3. Cancer Res. 2019; 79: 4612-26.

18. Zhang X, Shi H, Yao J, Li Y, Gao B, Zhang Y, et al. FAM225A facilitates colorectal cancer progression by sponging miR-613 to regulate NOTCH3. Cancer Med. 2020; 9: 4339-49.

19. J C, Y Y, H L, Q H, X C, Y H, et al. Long non-coding RNA PVT1 promotes tumor progression by regulating the miR-143/HK2 axis in gallbladder cancer. Molecular cancer. 2019; 18: 33.

20. Tian L, Yang L, Zheng W, Hu Y, Ding P, Wang Z, et al. RNA sequencing of exosomes revealed differentially expressed long noncoding RNAs in early-stage esophageal squamous cell carcinoma and benign esophagitis. Epigenomics. 2020; 12: 525-41.

21. Zong MZ, Feng WT, Du N, Yu XJ, Yu WY. Upregulation of long noncoding RNA LEF1-AS1 predicts a poor prognosis in patients with esophageal squamous cell carcinoma. Eur Rev Med Pharmacol Sci. 2019; 23: 7929-34.

22. Balas MM, Johnson AM. Exploring the mechanisms behind long noncoding RNAs and cancer. Noncoding RNA Res. 2018; 3: 108-17.

23. Liu W, Zheng J, Dong J, Bai R, Song D, Ma X, et al. Association of miR-197-5p, a Circulating Biomarker for Heart Failure, with Myocardial Fibrosis and Adverse Cardiovascular Events among Patients with Stage C or D Heart Failure. Cardiology. 2018; 141: 212-25.

24. Jain N, Roy J, Das B, Mallick B. miR-197-5p inhibits sarcomagenesis and induces cellular senescence via repression of KIAA0101. Mol Carcinog. 2019; 58: 1376-88.

25. Y J, T W, W L, R Z, M C. Circular RNA hsa_circ_0002024 suppresses cell proliferation, migration, and invasion in bladder cancer by sponging miR-197-3p. American journal of translational research. 2019; 11: 1644-52.

26. Li S, Kuhne WW, Kulharya A, Hudson FZ, Ha K, Cao Z, et al. Involvement of p54(nrb), a PSF partner protein, in DNA double-strand break repair and radioresistance. Nucleic Acids Res. 2009; 37: 6746-53.

27. Barboro P, Rubagotti A, Orecchia P, Spina B, Truini M, Repaci E, et al. Differential proteomic analysis of nuclear matrix in muscle-invasive bladder cancer: potential to improve diagnosis and prognosis. Cell Oncol. 2008; 30: 13-26.

28. Schiffner S, Zimara N, Schmid R, Bosserhoff AK. p54nrb is a new regulator of progression of malignant melanoma. Carcinogenesis. 2011; 32: 1176-82.

29. Ishiguro H, Uemura H, Fujinami K, Ikeda N, Ohta S, Kubota Y. 55 kDa nuclear matrix protein (nmt55) mRNA is expressed in human prostate cancer tissue and is associated with the androgen receptor. Int J Cancer. 2003; 105: 26-32.

30. Cheng R, Zhu S, Guo S, Min L, Xing J, Guo Q, et al. Downregulation of NONO induces apoptosis, suppressing growth and invasion in esophageal squamous cell carcinoma. Oncol Rep. 2018; 39: 2575-83. 